\title{
DIFFERENTIAL OPERATORS ON SOME SINGULAR SURFACES
}

\author{
R. HART AND S. P. SMITH
}

\begin{abstract}
Let $X$ be an irreducible affine algebraic variety of dimension 2 defined over an algebraically closed field of characteristic zero. Suppose that the normalisation $\bar{X}$ of $X$ is non-singular, and the natural projection $\pi: \tilde{X} \rightarrow X$ is injective. Further, suppose that $X$ is Cohen-Macaulay. Then the rings of differential operators $\mathscr{D}(X)$ and $\mathscr{D}(\tilde{X})$ are Morita equivalent.
\end{abstract}

\section{Introduction}

In [6] it is proved that if $X$ is an irreducible affine algebraic curve defined over an algebraically closed field of characteristic zero, and if $\pi: \tilde{X} \rightarrow X$, the projection from the normalisation, is injective, then the rings of differential operators $\mathscr{D}(X)$ and $\mathscr{D}(\tilde{X})$ are Morita equivalent. This paper is concerned with extending that result to higher dimensional varieties.

Throughout $k$ will be an algebraically closed field of characteristic zero, and $X$ denotes an irreducible affine algebraic variety over $k$. Denote the normalisation of $X$ by $\tilde{X}$ and let $\pi: \tilde{X} \rightarrow X$ be the projection. The ring of regular functions on $X$ is written $\mathcal{O}(X)$, and $\mathscr{D}(X)$ denotes the ring of differential operators on $X$ (see [6, section 1] for the definition). We will prove the following:

THEOREM. Let $X$ be a surface (that is $\operatorname{dim} X=2)$. Suppose that $\tilde{X}$ is non-singular and that $\pi: \tilde{X} \rightarrow X$ is injective. If $\mathcal{O}(X)=\bigcap\left\{\mathcal{O}(X)_{P} \mid\right.$ height $\left.P=1\right\}$ (or equivalently, $X$ is Cohen-Macaulay) then $\mathscr{D}(X)$ and $\mathscr{D}(\tilde{X})$ are Morita equivalent.

For background to this theorem the reader is referred to [6]. The proof of this theorem is along the same lines as that of [6, Theorem 3.4] where the analogous result for curves is proved. The $\mathscr{D}(X)-\mathscr{D}(\tilde{X})$ bimodule giving the Morita equivalence is again $\mathscr{D}(\tilde{X}, X)=\{\mathscr{D} \in \mathscr{D}(\tilde{X}) \mid D(f) \in \mathcal{O}(X)$ for all $f \in \mathcal{O}(\tilde{X})\}$. When $X$ satisfies the hypotheses of the theorem then $\operatorname{gl} . \operatorname{dim} \mathscr{D}(\widetilde{X})=2$, and so some work is required to show that $\mathscr{D}(\tilde{X}, X)$ is a projective right ideal of $\mathscr{D}(\tilde{X})$. In fact, if $X$ satisfies all the hypotheses of the theorem except the dimension condition, we are able to show that $\mathscr{D}(\tilde{X}, X)$ is a reflexive right ideal of $\mathscr{D}(X)$, and the requirement that $\operatorname{dim} X=2$ is made simply to ensure that any reflexive right ideal of $\mathscr{D}(\tilde{X})$ is projective. The other ingredient required to prove the theorem is to show that $\mathscr{D}(X)=\operatorname{End}_{\mathscr{D}(\tilde{X})} \mathscr{D}(\tilde{X}, X)$. This is proved 'locally' at the height 1 primes, and the intersection condition is required to give the global equality.

It is well-known that a Cohen-Macaulay ring satisfies the intersection condition; suppose the intersection condition is satisfied and consider a principal ideal $\alpha A$ in

Received 2 June 1986.

1980 Mathematical Subject Classification $14 \mathrm{~J} 17$.

Bull. London Math. Soc. 19 (1987) 145-148 
$A=\mathcal{O}(X)$. If $P$ is a height 1 prime in $A, \alpha A_{P}$ is a primary ideal in $A_{P}$. Using the intersection condition, it now follows easily that $\alpha A$ is an intersection of primary ideals for height 1 primes, so $\alpha A$ is unmixed. Therefore $A$ (being 2-dimensional) is Cohen-Macaulay.

It is clear that large parts of the proof will work for any $X$ satisfying all hypotheses of the theorem except that on $\operatorname{dim} X$, and one is naturally led to conjecture that there is a version of the theorem for $X$ of arbitrary dimension. We leave this as a problem, in the hope that the reader is more perceptive than the authors.

ACKNOWLedgement. The second author was supported by a British SERC Research Assistantship, and he would like to thank the SERC for its support.

\section{Proof of the Theorem}

The result is proved locally. For the moment let $\operatorname{dim} X \geqslant 1$, set $A=\mathcal{O}(X)$ and let $P$ be a height 1 prime of $A$. Write $B=A_{P}$, and $\bar{B}$ for the integral closure of $B$ in its field of fractions $K=$ Fract $B$. All the action takes place inside $\mathscr{D}(K)$, the space of $k$-linear differential operators on $K$. Some small modifications to the notation in [6] are required. As in $[6, \S 2.3], \mathscr{D}(\bar{B}, B)$ denotes the space of $k$-linear differential operators from $\bar{B}$ to $B$. Also, $\mathscr{D}(\bar{B}, B) * \bar{B}$ denotes the set of values of functions in $\mathscr{D}(\bar{B}, B)$. If $F \supseteq k$ is a field contained in $B$ we may view $B$ as an $F$-algebra, and we will write $\mathscr{D}^{F}(\bar{B}), \mathscr{D}^{F}(B)$, And $\mathscr{D}^{F}(\bar{B}, B)$ for the spaces of $F$-linear differential operators on $B$ etc. The key step in proving the theorem is the following (which has been independently established by $M$. Chamarie, with a similar proof).

Proposition 1. Let $X$ be an irreducible affine variety with $\operatorname{dim} X \geqslant 1$. Suppose that $\tilde{X}$ is smooth, and $\pi: \tilde{X} \rightarrow X$ is injective. For any height 1 prime $P$ of $A=\mathcal{O}(X), \mathscr{D}\left(\bar{A}_{P}, A_{P}\right) * \bar{A}_{P}=A_{P}$.

Proof. Set $n=\operatorname{dim} X$. Let $Q$ be the unique prime of $\mathcal{O}(X)$ lying over $P$. Write $B=A_{P}$ and $\bar{B}=\bar{A}_{P}=(\bar{A})_{P}$. Then $\bar{B}$ is a local ring with maximal ideal $Q \bar{B}$; this observation uses the lying-over and going-up properties of an integral extension. Let $m, M$ be the maximal ideals of the 1-dimensional local rings $B, \bar{B}$. Let $t \in M$ be a local parameter for the regular local ring $\bar{B}$.

The injectivity of $\pi$ ensures that the induced morphism $\operatorname{Spec} \bar{A} / Q \rightarrow \operatorname{Spec} A / P$ is injective, and hence birational [4, Theorem 4.6]. So $B / m=\operatorname{Fract}(A / P)=$ Fract $(\bar{A} / Q)=\bar{B} / M$. By Noether normalisation there is a polynomial ring $k\left[t_{1}, \ldots, t_{n}\right] \subseteq A$ with $A$ an integral extension. Since height $P=1$, we may assume that $k\left[t_{1}, \ldots, t_{n-1}\right] \cap P=0$. Set $F=k\left(t_{1}, \ldots, t_{n-1}\right)$ the field of rational functions. It follows that $B / m$ is a separable algebraic extension of $F$. Now consider $B$ and $\bar{B}$ as $F$-algebras.

By [5, Theorem 58] there is an exact sequence involving the modules of Kähler differentials:

$$
M / M^{2} \rightarrow \Omega_{\bar{B} / F} \otimes_{\bar{B}}(\bar{B} / M) \rightarrow \Omega_{(\bar{B} / M) / F} \rightarrow 0,
$$

where $d: \bar{B} \rightarrow \Omega_{\bar{B} \mid F}$ is the universal $F$-derivation and the first map is given by $t \rightarrow d t \otimes 1$. Because $\bar{B} / M$ is a separable algebraic extension of $F, \Omega_{(\bar{B} / M) / F}=0$. It follows that $\Omega_{\bar{B} / F}$ is free on $d t$. Let $\partial \in \operatorname{Der}_{F} \bar{B}$ be the $F$-linear derivation with $\partial(t)=1$; that is, $\partial=h d$, where $k: \Omega_{\bar{B} / F} \rightarrow \bar{B}$ is the $\bar{B}$-homomorphism with $h(d t)=1$.

Sublemma. $\mathscr{D}^{F}(\bar{B})[\partial]$ is a simple hereditary ring. 
Proof of sublemma. Since $\Omega_{\bar{B} / F}$ is free on $d t,[2, \S 16.11 .12]$ gives $\mathscr{D}^{F}(\bar{B})=\bar{B}[\partial]$. The simplicity is clear since $\bar{B}$ is a 1-dimensional regular local ring whose only ideals are $\bar{B} t^{n}$, and then the global dimension is given by [3, Theorem 2.6] for example.

Since $\bar{B} / B$ is a finitely generated torsion $B$-module, there exists $r \in \mathbb{N}$ with $M^{r} \subseteq B$. Consider $B / M^{r} \subseteq \bar{B} / M^{r}$. Since $B / M^{r}$ is local artinian, it is a complete local ring, and by Cohen's Theorem [5, Theorem 60] it contains a copy of its residue field. Let this copy be $L$; we can assume that $F \subseteq L$. Then $\bar{B} / M^{r}=L \oplus L s \oplus \ldots \oplus L s^{r-1}$ where $s$ is the image of $t$. Notice that $t \partial * M^{r} \subseteq M^{r}$, so we may consider $t \partial$ as a derivation on $\bar{B} / M^{r}$. Furthermore, since $L$ is a separable algebraic extension of $F$, and $t \partial$ is $F$-linear, $t \partial$ is also $L$-linear. Put $D=(t \partial-1) \ldots(t \partial-r+1) \in \mathscr{D}^{F}(\bar{B})$, and consider $D$ acting on $\bar{B} / M^{r}$. Then $D *\left(\bar{B} / M^{r}\right)=L \subseteq B / M^{r}$. Thus, lifting back to $\bar{B}, D * \bar{B} \subset B$, and $D * \bar{B} \notin m$. Hence $\mathscr{D}^{F}(\bar{B}, B) * \bar{B} \ddagger m$ and we must have $\mathscr{D}^{F}(\bar{B}, B) * \bar{B}=B$. This is true for the $F$-linear differential operators from $\bar{B}$ to $B$, so we certainly have the Proposition true for the $k$-linear operators.

Corollary 2. With the hypotheses of Proposition 1 , and $F$ as in the proof of Proposition $1, \mathscr{D}^{F}\left(A_{P}\right)$ and $\mathscr{D}^{F}\left(\bar{A}_{P}\right)$ are Morita equivalent. In particular $\mathscr{D}^{F}\left(A_{P}\right)$ is a simple hereditary ring.

Proof. In the proof of Proposition 1, it was seen that $\mathscr{D}^{F}\left(\bar{A}_{P}, A_{P}\right) * \bar{A}_{P}=A_{P}$, and that $\mathscr{D}^{F}\left(\bar{A}_{P}\right)$ is a simple hereditary ring. The Morita equivalence now follows exactly as in [6, Proposition 3.3], and hence $\mathscr{D}^{F}\left(A_{P}\right)$ has the properties stated.

Corollary 3. With the hypotheses of Proposition $1, \mathscr{D}\left(\bar{A}_{P}, A_{P}\right)$ is a projective right ideal of $\mathscr{D}\left(\vec{A}_{P}\right)$.

Proof. Since $\mathscr{D}^{F}\left(A_{P}\right)$ is a simple ring by the previous Corollary, and

$$
\mathscr{D}^{F}\left(\bar{A}_{P}, A_{P}\right) \mathscr{D}^{F}\left(A_{P}, \bar{A}_{P}\right)
$$

is a two-sided ideal of $\mathscr{D}^{F}\left(A_{P}\right)$, we must have $1 \in \mathscr{D}^{F}\left(\bar{A}_{P}, A_{P}\right) \mathscr{D}^{F}\left(A_{P}, \bar{A}_{P}\right)$; it follows that $1 \in \mathscr{D}\left(\bar{A}_{P}, A_{P}\right) \mathscr{D}\left(A_{P}, \bar{A}_{P}\right)$. Hence by the Dual Basis Lemma, $\mathscr{D}\left(\bar{A}_{P}, A_{P}\right)$ is a projective right $\mathscr{D}\left(\bar{A}_{P}\right)$-module.

CoRollary 4. Suppose that $X$ satisfies the hypotheses of Proposition 1, and in addition $\mathcal{O}(X)$ satisfies $\mathcal{O}(X)=\cap\left\{\mathcal{O}(X)_{P} \mid\right.$ height $\left.P=1\right\}$. Then $\mathscr{D}(\tilde{X}, X)$ is a reflexive right ideal of $\mathscr{D}(\tilde{X})$.

Proof. Write $I=\mathscr{D}(\tilde{X}, X)=\mathscr{D}(\bar{A}, A)$, and regard

$$
I^{* *}=\operatorname{Hom}_{\mathscr{D}(\tilde{X})}\left(\operatorname{Hom}_{\mathscr{D}(\tilde{X})}(I, \mathscr{D}(\tilde{X})), \mathscr{D}(\tilde{X})\right)
$$

as a right ideal of $\mathscr{D}(\tilde{X})$ containing $I$. Let $P$ be a height 1 prime of $A=\mathscr{O}(X)$. Note that $\left(I_{P}\right)^{*}=\left(I^{*}\right)_{P}$ and that $I_{P}=\mathscr{D}\left(\bar{A}_{P}, A_{P}\right)$ is a reflexive $\mathscr{D}\left(\bar{A}_{P}\right)$-module, by Corollary 3. Thus $I_{P}=\left(I_{P}\right)^{* *}=\left(I^{*}\right)_{P}^{*}=\left(I^{* *}\right)_{P}$ and so $\left(I^{* *}\right) * \bar{A} \subseteq\left(I^{* *}\right)_{P} * \bar{A}_{P}=I_{P} * \bar{A}_{P} \subseteq A_{P}$. But by the intersection hypothesis, $A=\bigcap A_{P}$, and so $\left(I^{* *}\right) * \bar{A} \subseteq A$, whence $I^{* *} \subseteq \mathscr{D}(\bar{A}, A)$ giving the equality $I^{* *}=I$ as required. 
The following observation was pointed out to the authors by J. T. Stafford. We would like to thank him for allowing us to include it here and so provide the final step in the proof of the theorem.

COROLlaRY 5. Under the hypothesis of Proposition 1, and the assumption that $\mathcal{O}(X)=\bigcap\left\{\mathcal{O}(X)_{P} \mid\right.$ height $\left.P=1\right\}$ it follows that $\mathscr{D}(X)=\operatorname{End}_{\mathscr{D}(\tilde{X})} \mathscr{D}(\tilde{X}, X)$.

Proof. Set $A=\mathcal{O}(X)$, and let $P$ be a height 1 prime. Since $\mathscr{D}\left(\bar{A}_{P}, A_{P}\right) * \bar{A}_{P}=A_{P}$ by Proposition 1, [6, Proposition 3.3] gives $\mathscr{D}\left(A_{P}\right)=\operatorname{End}_{\mathscr{D}\left(\bar{A}_{P}\right)} \mathscr{D}\left(\bar{A}_{P}, A_{P}\right)$. Since $A=\bigcap\left\{A_{P} \mid\right.$ height $\left.P=1\right\}$, we have $\bigcap_{\text {htP-1 }} \mathscr{D}\left(A_{P}\right) \subseteq \mathscr{D}(A)$ while the opposition inclusion is trivially true. Thus $\mathscr{D}(A)=\bigcap_{\mathrm{nt} P-1} \mathscr{D}\left(A_{P}\right)$. Therefore

$$
\mathscr{D}(A)=\bigcap_{\mathrm{nt}} \operatorname{End}_{\mathscr{D}\left(\bar{A}_{P}\right)} \mathscr{D}\left(\bar{A}_{P}, A_{P}\right) \supseteq \operatorname{End}_{\mathscr{D}(\bar{A})} \mathscr{D}(\bar{A}, A) \supseteq \mathscr{D}(A),
$$

and the result follows.

Proof of the Theorem. So now $\operatorname{dim} X=2$, and $\mathcal{O}(X)=\bigcap\left\{\mathcal{O}(X)_{P} \mid\right.$ height $\left.P=1\right\}$. By Corollary $5, \mathscr{D}(X)=\operatorname{End}_{\mathscr{D}(\tilde{X})} \mathscr{D}(\tilde{X}, X)$. By Corollary $4, \mathscr{D}(X, X)$ is a reflexive right ideal of $\mathscr{D}(\tilde{X})$. But as $\tilde{X}$ is non-singular and $\operatorname{dim} \tilde{X}=2$ we have $\operatorname{gl} \cdot \operatorname{dim} \mathscr{D}(\tilde{X})=2$ and so $\mathscr{D}(\tilde{X}, X)$ is actually projective [1, Proposition 5.2]. Since $\mathscr{D}(\tilde{X})$ is simple, $\mathscr{D}(\tilde{X}, X)$ is certainly a generator. Hence the result.

COROllary 6. With the hypotheses of the Theorem. $\mathscr{D}(\tilde{X}, X) * \mathcal{O}(\tilde{X})=\mathcal{O}(X)$.

Proof. Because $\mathscr{D}(X)$ is a simple ring, $\mathcal{O}(X)$ is a simple left $\mathscr{D}(X)$-module. Since $\mathscr{D}(\tilde{X}, X) * \mathscr{O}(\tilde{X})$ is a left $\mathscr{D}(X)$-module the result follows.

REMARK. If $X$ satisfies the hypotheses of the theorem then each irreducible component of $\operatorname{Sing} X$ is of codimension 1 in $X$. Recall [6, Proposition 7.2] that, if $X$ is singular but regular in codimension 1 (with $\tilde{X}$ smooth and $\pi$ injective), then $\mathscr{D}(X)$ is not simple.

\section{References}

1. H. BASs, 'Finitistic dimension and a homological generalisation of semi-primary rings', Trans. Amer. Math. Soc. 95 (1960) 466-488.

2. A. Grothendieck, Eléments de géométrie algébrique, Publ. Math. 32 (IHES, Paris, 1967).

3. R. Hart, 'Krull dimension and global dimension of simple Ore extensions', Math. Z. 121 (1971) 341-345.

4. J. HUMPHREYS, Linear algebraic groups (Springer, New York, 1977).

5. H. Matsumura, Commutative algebra (Benjamin, New York, 1970).

6. S. P. Smith and J. T. Stafford, 'Differential operators on an affine curve', preprint, University of Warwick, 1985.

School of Mathematics University of Leeds Leeds LS2 9JT
Mathematics Institute
University of Warwick
Coventry CV4 7AL

Current address of S. P. Smith:

Department of Mathematics

University of Washington

Seattle

WA 98195. L'S.A 